\title{
Identification and Molecular Characterization of the Stanniocalcin Family Gene from the Inshore Hagfish, Eptatretus burgeri
}

\section{Sekiguchi, Toshio}

Department of Bioscience and Biotechnology, Graduate School of Bioresource and Bioenvironmental Sciences, Kyushu University | Noto Marine Laboratory, Institute of Nature and Environmental Technology, Division of Marine Environmental Studies, Kanazawa University

\section{Kuraku, Shigehiro}

Department of Bioscience and Biotechnology, Graduate School of Bioresource and Bioenvironmental Sciences, Kyushu University | Phyloinformatics Unit, RIKEN Center for Life Science Technologies

\section{Tatsumi, Kaori}

Department of Bioscience and Biotechnology, Graduate School of Bioresource and Bioenvironmental Sciences, Kyushu University | Phyloinformatics Unit, RIKEN Center for Life Science Technologies

Shimasaki, Yohei

Department of Bioscience and Biotechnology, Graduate School of Bioresource and Bioenvironmental Sciences, Kyushu University | Laboratory of Marine Environmental Science, Faculty of Agriculture, Kyushu University

他

https://doi.org/10.5109/1800841

出版情報: 九州大学大学院農学研究院紀要. 62 (1)，pp.93-98，2017-02-24. Faculty of Agriculture， Kyushu University

バージョン :

権利関係 : 


\title{
Identification and Molecular Characterization of the Stanniocalcin Family Gene from the Inshore Hagfish, Eptatretus burgeri
}

\author{
Toshio SEKIGUCHI ${ }^{1 *}$, Shigehiro KURAKU ${ }^{2}$, Kaori TATSUMI ${ }^{2}$, Yohei SHIMASAKI ${ }^{3}$, \\ Yuji OSHIMA ${ }^{3}$ and Nobuo SUZUKI ${ }^{1}$
}

\author{
Department of Bioscience and Biotechnology, Graduate School of Bioresource and \\ Bioenvironmental Sciences, Kyushu University, Hakozaki 6-10-1, \\ Higashi-ku, Fukuoka, 812-8581, Japan \\ (Received October 28, 2016 and accepted November 4, 2016)
}

\begin{abstract}
Stanniocalcin (STC) is a glycoprotein hormone that was first isolated from teleost corpuscles of Stannius. $\mathrm{STC}$ is involved in the decrease in plasma $\mathrm{Ca}^{2+}$ level in teleosts. Comparative studies between invertebrate chordate STC and two paralogous vertebrate STCs suggest that the chordate ancestral gene of STC diverged into two paralogous genes in the evolution of vertebrates. However, this evolutionary scenario remains unclear. To clarify the diversification process of vertebrate STC, we made an attempt to isolate orthologs of STC in extant jawless fishes (cyclostomes) located most distantly in the vertebrate tree of life. In the present study, we searched the STC-like sequence by performing degenerate PCR in the inshore hagfish Eptatretus burgeri. We identified a STC homolog, designated as Eb-STC. Our molecular phylogenetic analysis confirmed that Eb-STC is a member of the STC family. The comparison of amino acid sequences revealed that Eb-STC is similar to gnathostome STC1 and 2, in that Eb-STC shares the 10 conserved cysteine residues that form intramolecular disulfide bonds. Eb-STC and gnathostome STCs possess the conserved $N$-glycosylated site. However, typical features discriminated between STC1 and 2, such as the distinct position of the cysteine residue associated with the intermolecular dimer, were not observed in Eb-STC. Tissue distribution analysis revealed that Eb-STC is expressed in various organs, but not in the brain. This expression pattern resembles that of teleost STC1 and 2, suggesting that Eb-STC acts as a paracrine factor. To the best of our knowledge, this is the first report on the identification and molecular characterization of STC in cyclostomes.
\end{abstract}

Key words: stanniocalcin, hagfish, cyclostomes, calcium homeostasis

\section{INTRODUCTION}

Stanniocalcin (STC) is a glycoprotein hormone first isolated from teleosts (Wagner and Dimattia, 2006; Wagner et al., 1992; Wagner et al., 1988; Wagner et al., 1986). In teleosts, an increase in the plasma calcium level elicits the release of STC from the corpuscles of Stannius (CS) (Wagner et al., 1998; Wagner and Jaworski, 1994; Wagner et al., 1991), which induces a decrease in the plasma $\mathrm{Ca}^{2+}$ level via the gill and intestine. In gills, STC stimulates the excretion of $\mathrm{Ca}^{2+}$ (Lafeber et al., 1988a; Lafeber et al., 1988b; Wagner et al., 1986). The chum salmon STC inhibits the $\mathrm{Ca}^{2+}$ uptake in perfused Atlantic cod intestine (Sundell et al., 1992). STC is also associated with phosphate metabolism. Treatment with coho salmon STC results in stimulation of inorganic phosphate $(\mathrm{Pi})$ reabsorption in the primary cultured flounder renal proximal tube cells (Lu et al., 1994). To date, STC

Noto Marine Laboratory, Institute of Nature and Environmental Technology, Division of Marine Environmental Studies, Kanazawa University, Housu-gun, Ishikawa 927-0553, Japan

2 Phyloinformatics Unit, RIKEN Center for Life Science Technologies, 2-2-3 Minatojima-minami, Chuo-ku, Kobe, 6500047, Japan

3 Laboratory of Marine Environmental Science, Faculty of Agriculture, Kyushu University, Hakozaki 6-10-1, Higashi-ku, Fukuoka 812-8581, Japan

* Corresponding author (E-mail: t-sekiguchi@se.kanazawa-u. ac.jp) has been observed in a wide range of species including vertebrates, invertebrate deuterostomes, protostomes, and unicellular eukaryotes (Roch and Sherwood, 2010; 2011; Schein et al., 2012). The paralogous genes of STC1 and STC2 have been identified in various organisms, from teleosts to mammals (Luo et al., 2005; Shin and Sohn, 2009). Studies of vertebrate STC have uncovered its molecular characteristics. The vertebrates STC1 and STC2 have in common the $N$-glycosylated site and contain 10 cysteine residues forming five intramolecular disulfide bonds (Hulova and Kawauchi, 1999). Additionally, they form a homodimer via an intermolecular disulfide bond that is located in a different position in each STC paralog (Roch and Sherwood, 2011). In vertebrate STC1, the conserved 11th cysteine is involved in the formation of a homodimer (Hulova and Kawauchi, 1999). On the other hand, vertebrate STC2 shares the 3 conserved cysteine residues, one of which is expected to form an intermolecular disulfide bond (Roch and Sherwood, 2011). Furthermore, a histidine-rich sequence is conserved in vertebrate STC2 (Roch and Sherwood, 2011). Comparisons of the sequences of STC in invertebrate chordates including ascidian and amphioxus demonstrate that the STCs possess prototypical features of vertebrate STC1 and 2, implying that vertebrate STC1 and 2 are derived from an ancestral STC of primitive chordates (Roch and Sherwood, 2010). To obtain insight into the more precise evolutionary process, we focused on the cyclostomes including hagfish and lamprey. Cyclostomes are jawless vertebrates and 
phylogenetically located in the basal position in vertebrates (Kuraku, 2013; Shimeld and Donoghue, 2012). However, no STC gene has been identified in cyclostomes.

In the present study, to elucidate the evolutional diversity of vertebrate STC1 and 2, we explored the STC gene from the inshore hagfish, Eptatretus burgeri. We cloned and determined the sequence of the STC-like gene, designated as $E$. burgeri STC (Eb-STC). Furthermore, its molecular characteristics and tissue distribution pattern were clarified.

To the best of our knowledge, this is the first identification of STC in cyclostomes.

\section{MATERIALS AND METHODS}

\section{Animals}

Inshore hagfish (E. burgeri) were obtained from near the Noto-Jima Island by a fisherman. All experimental procedures were conducted in accordance with the Guide for the Care and Use of Laboratory Animals prepared by the Kanazawa University.

\section{Molecular cloning of STC}

The digestive tract was removed from adult hagfish. First-strand cDNA was synthesized using the SuperScript $^{\circledR}$ III reverse transcriptase (Thermo Fisher Scientific, Waltham, MA, USA) and oligo- $(\mathrm{dT})_{20}$ primer following extraction of total RNA. The 371-bp fragment of putative STC cDNA was obtained by degenerate reverse transcription polymerase chain reaction (RT-PCR) using the forward primer 5'-SANGYNGGITGYGSIDYITT-3' and the reverse primer 5'-TCITSIYCRCAIBYIADNA-3'. Additionally, we determined the open reading frame (ORF) of STC by 5' and 3'rapid amplification of cDNA ends (RACE) using the GeneRacer ${ }^{\circledR}$ kit (Thermo Fisher Scientific, Waltham, MA, USA). The cDNA library was synthesized from $2 \mu \mathrm{g}$ total RNA using the oligo(dT) adapter primer and was amplified using the adapter primer and the gene-specific complementary first primers (5'-RACE first primer: 5'-TTGAGGTGTTGGAAGGTGAGGGAGAATG-3'; 3'-RACE first primer: 5'GTGAAACGGGAGGTCTCCATGCCATCTG-3'). The first PCR product was amplified using the 5' or 3' nested adapter primer and each second gene-specific primer (5'-RACE second primer: 5'-CGCCACCGTCTCATCATCGCACTTAATC-3'; 3'-RACE second primer: 5'GGAGATGGTGGTACAACTGCAGCGTCAT-3'). The second PCR products were subcloned and sequenced by the ABI PRISM ${ }^{\mathrm{TM}} 3130$ Genetic Analyzer with a Big-Dye Sequencing Kit version 3.1 (Thermo Fisher Scientific) using the universal primers SP6 and T7.

\section{Molecular phylogenetic analysis of STC}

Molecular phylogenetic analysis was performed as described previously (Sekiguchi et al., 2016; Sekiguchi et al., 2009). In brief, the full-length amino acid sequences of chordate STC were aligned using the CLUSTAL X program (Higgins and Sharp, 1988), and the alignment was manually checked using BioEdit version 7.0.5.3. After the removal of gaps, phylogenetic trees were inferred using alignments by the neighbor-joining method with MEGA version 7.0 (Saitou and Nei 1987; Tamura et al., 2013). The accession numbers of the protein sequences used in this analysis are as follows: human STC1 (NP_003146), chicken STC1 (XP_425760), turkey STC1 (XP_010721360), platypus STC (ABI64157), American alligator STC1 (XP_006274704), pig STC1 (NP_001096682), cattle STC1 (NP_788842), mouse STC1 (AAP47156), coelacanth STC1 (XP_005991938), African clawed frog STC1 (AAH76749), green anole STC1 (XP_008118056), spotted gar STC-like (XP_006625371), bowfin STC (BAC66163), zebrafish STC1b (AAI22229), medaka STC1b (ENSORLP00000013724), stickleback STC1b (ENSGACP00000014284), zebrafish STC1a (AAH66540), medaka STC1a (ENSORLP00000000399), Japanese flounder STC1 (ACJ06521), elephant nose fish STC (BAD99600), ghost shark STC-like-a (SINCAMP00000001885), skate STC (FF600059), ghost shark STC-like-b (SINCAMP00000001117), Japanese flounder STC2 (ACJ06521), medaka STC2a (ENSORLP00000009199), stickleback STC2a (ENSGACP00000024367), zebrafish STC2a (NP_001014827), spotted gar STC2 (XP_006631778), coelacanth STC2 (XP_005992572), tropical clawed frog STC2 (AAI21339), cattle STC2 (XP_005899841), pig STC2 (NP_001103643), human STC2 (NP_003705), mouse STC2 (NP_035621), green anole STC2 (XP_008102897), chicken STC2 (XP_414534), turkey STC2 (XP_003210363), American alligator STC2 (XP_006270913), and ascidian (Ciona intestinalis) STC-like (XP_002130163).

\section{Reverse-transcription (RT)-PCR of STC and GAPDH mRNAs in various tissues in hagfish}

The brain, gill, heart, liver, gallbladder, digestive tract, and kidney of the hagfish were removed. Total RNA $(1 \mu \mathrm{g})$ was extracted and reverse-transcribed using the SuperScript ${ }^{\circledR}$ III reverse transcriptase (Thermo Fisher Scientific) and oligo- $(\mathrm{dT})_{20}$ primer. Next, we performed PCR of STC using the primer set GGTGTTCAACAGAACGGTGC (forward primer) and ATGACGCTGCAGTTGTACCA (reverse primer). PCR of GAPDH was performed as an internal control of each tissue using the primer set GTCTGGTGACCAGGGC (forward primer) and CAATCCCAAAGTTATCATGG (reverse primer). We checked the fragment size of PCR products by agarose gel electrophoresis. Moreover, sequences of the PCR fragments were directly determined by the ABI PRISM $^{\mathrm{TM}} 3130$ Genetic Analyzer, using specific PCR primers of STC and GAPDH. This experiment was independently performed in three individuals.

\section{RESULTS}

\section{Molecular characterization of the STC ortholog in hagfish}

To identify the orthologous gene of STC in hagfish, we cloned and determined the full-length ORF sequence of STC from a first-strand cDNA library of the digestive tract. First, the 371-bp sequence was determined by 


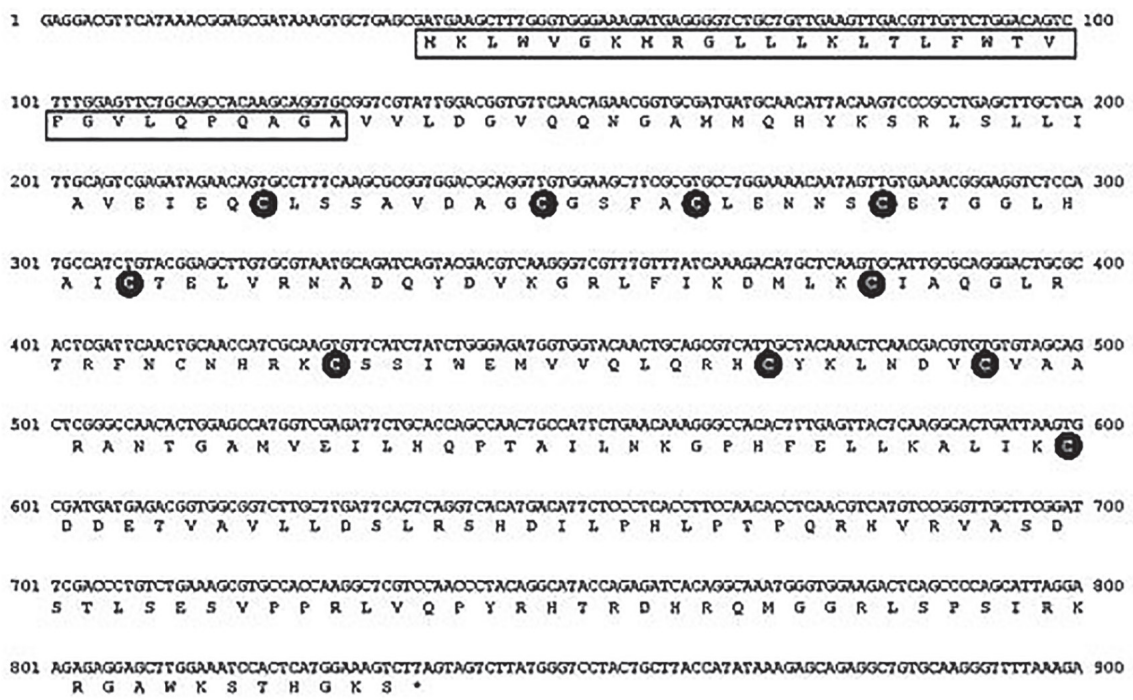

$902 \cos 904$

Fig. 1. Nucleotide and deduced amino acid sequences of hagfish STC.

The predicted signal peptide is shown by the open box. Black circles show cysteine residues observed in vertebrate STC. The stop codon is depicted by an asterisk. These data are available under GenBank accession no. LC191307.

PCR analysis using degenerated primers designed from conserved sequence stretches in gnathostome STC. Moreover, an ORF sequence of 801 bp was determined using the 5'- and 3'-RACE methods. The deduced amino acid sequence of 266 residues possessed the putative 31-amino acid signal peptide, which was predicted by the Signal IP4 server (Petersen et al., 2011) (Fig. 1). Ten cysteine residues are detected in this protein (Fig. 1).

In an attempt to elucidate whether this protein is orthologous to gnathostome STC, we performed molecular phylogenetic analysis. As shown in Fig. 2, the molecular phylogenetic tree of chordate STC demonstrated that the vertebrate STC family is divided into two groups, namely STC1 and STC2, with bootstrap values of 99 and 63 , respectively. The hagfish STC-like protein showed a higher proximity to STC2 and was placed outside the gnathostome sequences in accordance with the phylogenetic position of the hagfish (Fig. 2). Therefore, we designated this sequence as E. burgeri STC (Eb-STC).

In order to evaluate the molecular characteristics of Eb-STC, we compared the amino acid sequence of EbSTC with that of various chordate STCs. Multiple amino acid sequence alignment analyses of Eb-STC, gnathostome STCs, and ascidian STC demonstrated that 10 cysteine residues are conserved in STCs of all chordates (Fig. 3). Furthermore, the $N$-glycosylation site is conserved in vertebrate STC. However, the 11th cysteine residue that is responsible for dimer formation of gnathostome STC1 was not detected in Eb-STC (Fig. 3). Moreover, the three cysteine residues that are conserved in gnathostome STC2s were not observed in hagfish STC (Fig. 3). No gnathostome STC2-specific histidine-rich sequence was detected in hagfish STC (Fig. 3).

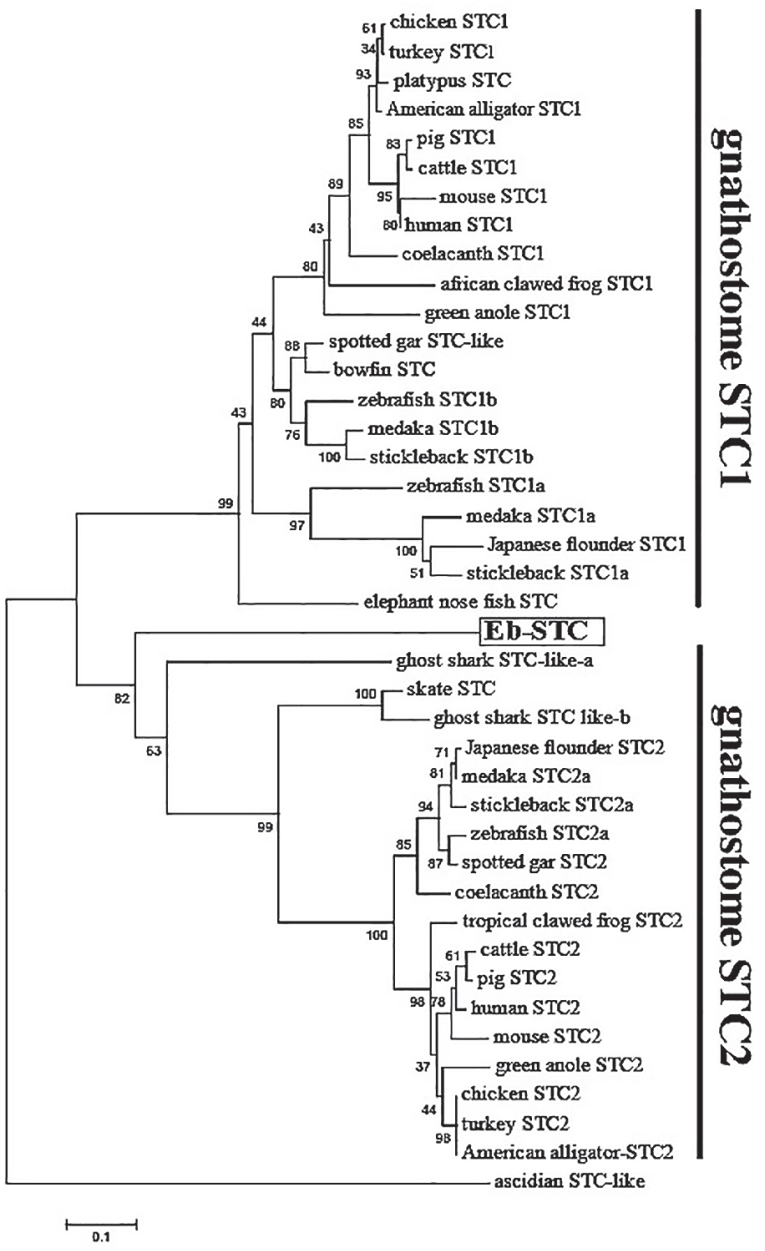

Fig. 2. Molecular phylogenetic tree of STC in chordates. Molecular phylogenetic tree prediction was performed using the neighbor-joining method. The bootstrap value is shown beside each branch. The unrooted tree is depicted as a root tree. The scale bar represents an evolutionary distance of 0.1 amino acid substitutions per protein. 

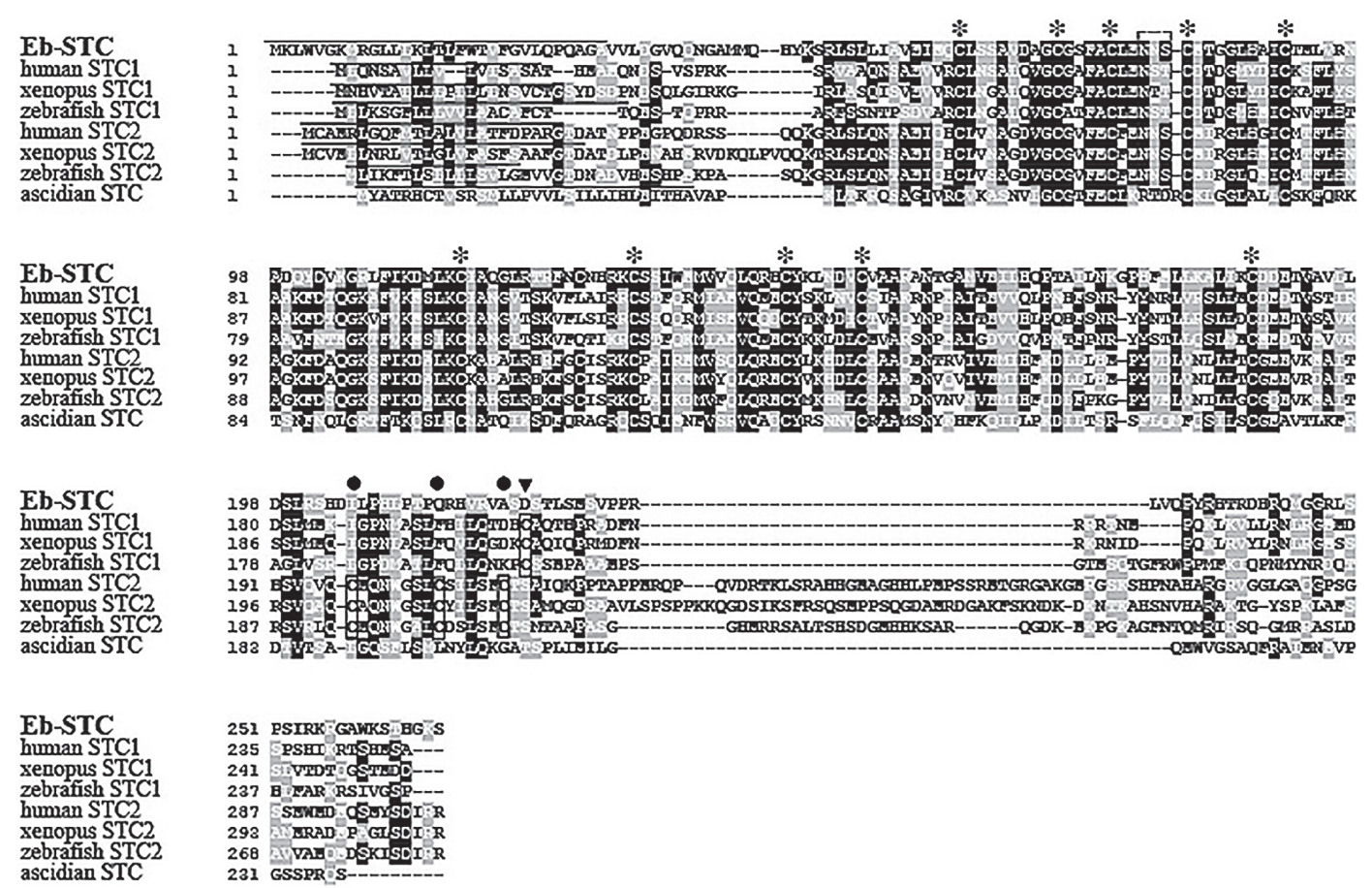

Fig. 3. Amino acid comparison of Eb-STC with chordate STC1 and 2.

Identical and similar amino acids among half of the total members are depicted by black and gray boxes, respectively. The 10 conserved cysteine residues among STCs are indicated by asterisks. The cysteine residue conserved in the gnathostome STC1s is indicated by an open box and arrowhead. The three cysteine residues conserved in the gnathostome STC2s are indicated by open boxes and closed black circles. The $N-$ glycosylation site is indicated by a dotted box. Signal peptides are shown by over bars. Signal peptides are shown in overbar.

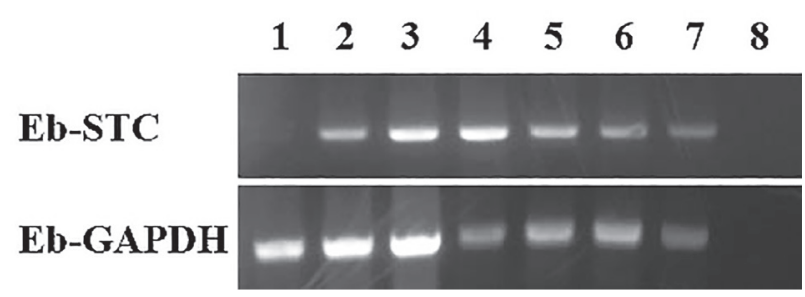

Fig. 4. RT-PCR analysis of Eb-STC mRNA.

The distribution of Eb-STC was examined using RT-PCR in the brain (lane 1), gill (lane 2), heart (lane 3), liver (lane 4), gallbladder (lane 5), digestive tract (lane 6), and kidney (lane 7). No product was detected in the absence of first-strand cDNA (DW, lane 8). Eb-GAPDH represents an internal control.

\section{Expression analysis of Eb-STC mRNA in various tissues}

To evaluate the tissue distribution pattern of $\mathrm{Eb}-$ STC, we performed RT-PCR analysis in various tissues of E. burgeri. The Eb-STC transcript was detected in the gill, heart, liver, gallbladder, digestive tract, and kidney (Fig. 4).

\section{DISCUSSION}

Plasma $\mathrm{Ca}^{2+}$ levels are stably maintained by various hormones, given that $\mathrm{Ca}^{2+}$ plays a central role in the physiological function of vertebrates (Norris and Carr, 2013). STC is a hormone involved in calcium homeosta- sis in teleosts and has been identified in various species (Roch and Sherwood, 2011). In the present study, we identified the STC gene from the hagfish $E$. burgeri and confirmed its orthology to the gnathostome STC family member and its molecular characteristics.

Hagfish STC named as Eb-STC possesses the signal peptide that is necessary for secreting peptides and 10 cysteine residues typically observed in STC (Fig. 1). Molecular phylogenetic analysis revealed that gnathostome STCs were divided into two clades as previously reported (Wagner and Dimattia, 2006). Eb-STC was placed in proximity to the clade formed by gnathostome STC2s, suggesting that Eb-STC is orthologous to gnathostome STC2. However, owing to limited information on cyclostomes and elasmobranchs, the possibility that $\mathrm{Eb}-\mathrm{STC}$ is the common ancestor of both gnathostome STC1 and STC2 cannot be ruled out. To uncover the precise phylogenetic position of Eb-STC, explorations of the Eb-STC paralogous gene in E. burgeri and the STC gene in lamprey are currently underway.

An amino acid sequence comparison among chordate STCs indicates that Eb-STC shares a common sequence with vertebrate STC. The $N$-glycosylation site and 10 cysteine residues are conserved among vertebrates including hagfish (Fig. 3) (Roch and Sherwood, 2011). On the other hand, the gnathostome STC1-specific 11th cysteine and three cysteine residues conserved in gnathostome STC2 were not detected in EbSTC (Fig. 3). In addition, no 11th cysteine residue was 
observed after the 10 conserved cysteine residues. These findings suggest that Eb-STC functions as a monomer, like arowana and ascidian (Amemiya et al., 2006; Roch and Sherwood, 2010). In amphioxus, two STC types were identified (Roch and Sherwood, 2010). One STC is presumed to act as a monomer, and the other STC is a putative homodimer type, indicating that the homodimerized type of STC is conserved in chordates. Taken together, these findings imply that hagfish also possesses the other STC gene forming the homodimer.

Tissue distribution analysis demonstrated ubiquitous expression of Eb-STC in all organs except the brain (Fig. 4). In bony fish, STC1 and 2 are commonly expressed in various tissues including the CS (Hang and Balment, 2005; Shin et al., 2006; Shin and Sohn, 2009). The expression pattern of euryhaline flounder STC is coincident with that of Eb-STC (Hang and Balment, 2005). Altogether, these findings imply that the tissue distribution pattern of Eb-STC is similar to that in gnathostomes. Moreover, it is suggested that Eb-STC acts as a paracrine factor. One possible function of Eb-STC is $\mathrm{Ca}^{2^{+}}$ion homeostasis. The hagfish is an osmoconformer. The osmotic pressure and univalent ion (such as $\mathrm{Na}^{+}, \mathrm{Cl}^{-}$, and $\mathrm{K}^{+}$) levels of their internal environment are the same as those of seawater. However, the $\mathrm{Ca}^{2+}$ and $\mathrm{Mg}^{2+}$ ion levels in hagfish serum are stably maintained at lower levels than in seawater (Sardella et al., 2009). The extract from the eel CS stimulates the release of $\mathrm{Ca}^{2+}$ from the gill of Eptatretus cirrhatus. These issues suggest that EbSTC induces $\mathrm{Ca}^{2+}$ release in the gill (Forster and Fenwick, 1994). Additionally, Pi metabolism is observed in the Pacific hagfish, Eptatretus stoutii, and the Pi transporter Slc34a is expressed in the gill, skin, intestine, and kidney (Schultz et al., 2014). Taking into account the involvement of STC on Pi homeostasis in teleosts (Lu et al., 1994), it is suggested that Eb-STC is also associated with Pi homeostasis. To uncover STC functions, further functional research is needed.

In the present study, we first identified the STC gene in cyclostomes. This study provides insight into the evolutionary biology of the STC gene.

\section{ACKNOWLEDGMENTS}

We are grateful to Mr. Yasuyuki Hirayama for providing the hagfish. This study was supported in part by grants to T.S. (Scientific Research [C] No. 15K07126 by JSPS) and to N.S. (Grant-in-Aid for Scientific Research [C] No. 24620004 by JSPS). This study was conducted as part of the cooperative research program of the Institute of Nature and Environmental Technology, Kanazawa University.

\section{REFERENCES}

Amemiya, Y., D. M. Irwin and J. H. Youson 2006 Cloning of stanniocalcin (STC) cDNAs of divergent teleost species: Monomeric STC supports monophyly of the ancient teleosts, the osteoglossomorphs. Gen. Comp. Endocrinol., 149: 100-107

Forster, M. E. and J. C. Fenwick 1994 Stimulation of calcium efflux from the hagfish, Eptatretus cirrhatus, gill pouch by an extract of corpuscles of Stannius from an eel (Anguilla dieffenbachii): teleostei. Gen. Comp. Endocrinol., 94: 92-103

Hang, X. and R. J. Balment 2005 Stanniocalcin in the euryhaline flounder (Platichthys flesus): primary structure, tissue distribution, and response to altered salinity. Gen. Comp. Endocrinol., 144: 188-195

Higgins, D. G. and P. M. Sharp 1988 CLUSTAL: a package for performing multiple sequence alignment on a microcomputer. Gene, 73: 237-244

Hulova, I. and H. Kawauchi 1999 Assignment of disulfide linkages in chum salmon stanniocalcin. Biochem. Biophys. Res. Commun., 257: 295-299

Kuraku, S. 2013 Impact of asymmetric gene repertoire between cyclostomes and gnathostomes. Semin. Cell Dev. Biol., 24: 119-127

Lafeber, F. P., G. Flik, S. E. Wendelaar Bonga and S. F. Perry 1988a Hypocalcin from Stannius corpuscles inhibits gill calcium uptake in trout. Am. J. Physiol., 254: R891-896

Lafeber, F. P., R. G. Hanssen, Y. M. Choy, G. Flik, M. P. HerrmannErlee, P. K. Pang and S. E. Bonga 1988b Identification of hypocalcin (teleocalcin) isolated from trout Stannius corpuscles. Gen. Comp. Endocrinol., 69: 19-30

Lu, M., G. F. Wagner and J. L. Renfro 1994 Stanniocalcin stimulates phosphate reabsorption by flounder renal proximal tubule in primary culture. Am. J. Physiol., 267: R1356-1362

Luo, C. W., M. D. Pisarska and A. J. Hsueh 2005 Identification of a stanniocalcin paralog, stanniocalcin-2, in fish and the paracrine actions of stanniocalcin-2 in the mammalian ovary. Endocrinology, 146: 469-476

Norris, D. O. and J. A. Carr 2013 Chapter 14 - Regulation of Calcium and Phosphate Homeostasis in Vertebrates. Vertebrate Endocrinology (Fifth Edition). Academic Press, San Diego, pp. 501-527

Petersen T. N., S. Brunak, G. von Heijne and H. Nielsen 2011 SignalP 4.0: discriminating signal peptides from transmembrane regions. Nature Methods 8: 785-786

Roch, G. J. and N. M. Sherwood 2010 Genomics reveal ancient forms of stanniocalcin in amphioxus and tunicate. Integr. Comp. Biol., 50: 86-97

Roch, G. J. and N. M. Sherwood 2011 Stanniocalcin has deep evolutionary roots in eukaryotes. Genome Biol. Evol., 3: 284-294

Saitou, N. and M. Nei 1987 The neighbor-joining method: a new method for reconstructing phylogenetic trees. Mol. Biol. Evol., 4: 406-425

Sardella, B. A., D. W. Baker and C. J. Brauner 2009 The effects of variable water salinity and ionic composition on the plasma status of the Pacific Hagfish (Eptatretus stoutii). J. Comp. Physiol. B, 179: 721-728

Schein, V., J. C. Cardoso, P. I. Pinto, L. Anjos, N. Silva, D. M. Power and A. V. Canario 2012 Four stanniocalcin genes in teleost fish: structure, phylogenetic analysis, tissue distribution and expression during hypercalcemic challenge. Gen. Comp. Endocrinol., 175: 344-356

Schultz, A. G., S. C. Guffey, A. M. Clifford and G. G. Goss 2014 Phosphate absorption across multiple epithelia in the Pacific hagfish (Eptatretus stoutii). Am. J. Physiol. Regul. Integr. Comp. Physiol., 307: R643-652

Sekiguchi, T., K. Kuwasako, M. Ogasawara, H. Takahashi, S. Matsubara, T. Osugi, I. Muramatsu, Y. Sasayama, N. Suzuki and H. Satake 2016 Evidence for Conservation of the Calcitonin Superfamily and Activity-regulating Mechanisms in the Basal Chordate Branchiostoma floridae: insights into the molecular and functional evolution in chordates. J. Biol. Chem., 291: $2345-2356$

Sekiguchi, T., N. Suzuki, N. Fujiwara, M. Aoyama, T. Kawada, K. Sugase, Y. Murata, Y. Sasayama, M. Ogasawara and H. Satake 2009 Calcitonin in a protochordate, Ciona intestinalis-the prototype of the vertebrate calcitonin/calcitonin gene-related peptide superfamily. FEBS J., 276: 4437-4447

Shimeld, S. M. and P. C. Donoghue 2012 Evolutionary crossroads in developmental biology: cyclostomes (lamprey and hagfish). Development, 139: 2091-2099 
Shin, J., D. Oh and Y. C. Sohn 2006 Molecular characterization and expression analysis of stanniocalcin-1 in turbot (Scophthalmus maximus). Gen. Comp. Endocrinol., 147: 214-221

Shin, J. and Y. C. Sohn 2009 cDNA cloning of Japanese flounder stanniocalcin 2 and its mRNA expression in a variety of tissues. Comp. Biochem. Physiol. A Mol. Integr. Physiol., 153: 24-29

Sundell, K., B. T. Bjornsson, H. Itoh and H. Kawauchi 1992 Chum salmon (Oncorhynchus keta) stanniocalcin inhibits in vitro intestinal calcium uptake in Atlantic cod (Gadus morhua). J. Comp. Physiol. B, 162: 489-495

Tamura, K., G. Stecher, D. Peterson, A. Filipski and S. Kumar 2013 MEGA6: Molecular Evolutionary Genetics Analysis version 6.O. Mol. Biol. Evol., 30: 2725-2729

Wagner, G. F. and G. E. Dimattia 2006 The stanniocalcin family of proteins. J. Exp. Zool. A Comp. Exp. Biol., 305: 769-780

Wagner, G. F., G. E. Dimattia, J. R. Davie, D. H. Copp and H. G. Friesen 1992 Molecular cloning and cDNA sequence analysis of coho salmon stanniocalcin. Mol. Cell. Endocrinol., 90: 7-15
Wagner, G. F., J. C. Fenwick, C. M. Park, C. Milliken, D. H. Copp and H. G. Friesen 1988 Comparative biochemistry and physiology of teleocalcin from sockeye and coho salmon. Gen. Comp. Endocrinol., 72: 237-246

Wagner, G F., M. Haddad, R. C. Fargher, C. Milliken and D. H. Copp 1998 Calcium is an equipotent stimulator of stanniocalcin secretion in freshwater and seawater salmon. Gen. Comp. Endocrinol., 109: 186-191

Wagner, G. F., M. Hampong, C. M. Park and D. H. Copp 1986 Purification, characterization, and bioassay of teleocalcin, a glycoprotein from salmon corpuscles of Stannius. Gen. Comp. Endocrinol., 63: 481-491

Wagner, G. F. and E. Jaworski 1994 Calcium regulates stanniocalcin mRNA levels in primary cultured rainbow trout corpuscles of stannius. Mol. Cell. Endocrinol., 99: 315-322

Wagner, G. F., C. Milliken, H. G. Friesen and D. H. Copp 1991 Studies on the regulation and characterization of plasma stanniocalcin in rainbow trout. Mol. Cell. Endocrinol., 79: 129138 